\title{
hOSPITAL DE CLÍNICAS DA UNICAMP: 30 ANOS DE HISTÓRIA CUIDANDO DA VIDA
}

\author{
HOSPITAL DE CLÍNICAS OF UNICAMP: \\ 30 YEARS OF HISTORY CARING FOR LIFE
}

Antonio Gonçalves Oliveira Filho ${ }^{1}$

\section{RESUMO}

Este artigo apresenta um pouco da história do Hospital de Clínicas da UNICAMP, desde a sua idealização para servir de hospital escola para a Faculdade de Ciências Médicas da UNICAMP no início dos anos setenta até os dias de hoje, quando o HC completa 30 anos de atividades no cuidado da população de Campinas e região, servindo também de palco para ensino e capacitando profissionais para atuação em várias áreas da saúde, bem como proporcionando um ambiente rico para importantes pesquisas na saúde. O HC da UNICAMP apresenta indicadores significantes em todas as categorias e se firma como um dos mais importantes elos de relacionamento da universidade com a sociedade.

PALAVRAS-CHAVE: Universidade. Hospital. Formação Profissional. Saúde Pública.

\footnotetext{
${ }^{1}$ Professor de Cirurgia Pediátrica do Departamento de Cirurgia da Faculdade de Ciências Médicas da Universidade Estadual de Campinas, UNICAMP. Mestre em Ciências da Cirurgia pela Universidade Estadual de Campinas (1992) e Doutor em Ciências da Cirurgia pela Universidade Estadual de Campinas (2003). Atualmente é Coordenador de Assistência do Hospital de Clínicas da UNICAMP. E-mail: oliveirag@uol.com.br
} 


\begin{abstract}
This paper shows a summary of the history of the Hospital of Clinics of UNICAMP, from its idealization to serve as a university hospital for the School of Medical Sciences of UNICAMP in the early seventies to the present day, when the HC completes 30 years of activities caring of the inhabitants of Campinas and the region, also serving as a place for teaching and training professionals to work in various health areas, as well as providing a rich environment for important health research. The HC of UNICAMP presents significant indicators in all categories and is recognized as one of the most important links of the university's relationship with society.
\end{abstract}

KEYWORDS: University. Hospital. Professional Training. Public Health.

Hospital de Clínicas da UNICAMP ou como é mais conhecido, HC da UNICAMP, foi idealizado pelo fundador da Universidade Estadual de Campinas, o senhor Zeferino Vaz, para ser o elo mais visível das relações da UNICAMP com a sociedade.

A Faculdade de Medicina no início de sua história exerceu suas atividades de ensino, pesquisa e assistência nos hospitais da Maternidade de Campinas e na Santa Casa de Misericórdia de Campinas, porém com os projetos de expansão da Universidade no início dos anos 70 e o aumento das demandas de ensino, especialização e pesquisa, ficou definido a construção do HC no campus da UNICAMP localizado no distrito de Barão Geraldo em Campinas, sendo lançada a pedra fundamental de sua construção em 1975.

Após o término da construção da primeira fase, os 53 consultórios dos ambulatórios foram inaugurados em fevereiro de 1979, os primeiros 195 colaboradores foram contratados e iniciou-se o movimento de pacientes que procuram cuidado para suas doenças e angústias, o qual veio aumentando muito ao longo desses 30 anos.

Com a conclusão das obras em 1985, no início de 1986 iniciaram-se as transferências e ocupação das enfermarias e em dezembro foi realizada a primeira 
cirurgia nas dependências do HC, passando-se assim toda a Assistência, Ensino e Pesquisa da Faculdade de Ciências Médicas - FCM - a ser realizada no novo hospital.

Ao longo desses 30 anos de história, o HC da UNICAMP com suas instalações, sua equipe de profissionais de saúde e administrativos, atendeu cerca de 6 milhões de pessoas procedentes de todo o país e também de outros países, realizou cerca de 400 mil cirurgias, 6.000 transplantes e foi palco de ensino/aprendizagem para cerca de 15 mil alunos de graduação de Medicina, Enfermagem, Farmácia e Fonoaudiologia. Passaram pelo Hospital cerca de 10 mil residentes de diversas especialidades os quais levaram os ensinamentos aqui aprendidos para várias regiões do Brasil e exterior, o que denota seu compromisso na formação de alto nível de pessoas qualificadas e preparadas para o atendimento da saúde da população.

Durante estes anos, o HC se consolidou como um hospital que presta atendimento de saúde terciário de alta complexidade, atendendo exclusivamente pacientes do Sistema Único de Saúde (SUS) e também que propõe ações de atendimento comunitário à sociedade através de projetos específicos e exitosos como o projeto catarata, ações de prevenção de diabetes e obesidade e diagnóstico de deficiência visual nas escolas primárias.

A história de assistência à saúde que o HC presta hoje, esteve sempre ligada à atuação do Serviço Social muito antes dele ter sido idealizado.

Há 50 anos, as assistentes sociais que atuavam na Maternidade de Campinas, juntamente com o professor Miguel Tobar Acosta criaram e estruturaram o que é hoje o Serviço Social do Hospital de Clínicas.

Nestes 50 anos de atuação, o Serviço Social do Hospital de Clínicas desempenhou o importante papel de promover a atenção e o cuidado aos pacientes que procuram assistência médica, identificar e lutar pelas pessoas que se apresentavam em situações de vulnerabilidade, bem como é um importante serviço que luta e trabalha pelo resgate da cidadania destes pacientes menos favorecidos através da busca de direitos que muitos deles desconhecem totalmente.

Consagrado pela assistência de qualidade, o HC, vem investindo cada vez mais na especialização de seus profissionais e na atualização de seu parque tecnológico para adequações às demandas modernas da assistência médica. Dez mil pessoas circulam 
diariamente pelo Hospital, boa parte em busca de atendimento de saúde, que compreende 44 especialidades clínicas, divididas em quase 600 subespecialidades médicas.

Atualmente, o Hospital ocupa uma área de $104.000 \mathrm{~m}^{2}$ e possui uma área construída de aproximadamente $65.000 \mathrm{~m}^{2}$, distribuídos em sete blocos interligados. Anualmente são realizados 2,6 milhões de consultas e procedimentos ambulatoriais, mais de 3,3 milhões de exames, 15 mil internações eletivas e de urgência e quase 15 mil cirurgias ${ }^{2}$.

O HC é referência nacional para vários tratamentos no Brasil como os implantes cocleares para crianças e adultos, as cirurgias para tratamento de epilepsia, as cirurgias para tratamento da obesidade e é o único Centro de Alta Complexidade para tratamento de Oncologia (CACON) da região. Hoje, os 195 colaboradores que foram contratados em 1979, são aproximadamente 2.500, aliados a aproximadamente 1.000 alunos, residentes, aprimorandos e professores atuantes no atendimento de altíssimo nível e humanizado à população que procura atendimento no Hospital de Clínicas.

A forte vocação para investigações clínicas e científicas das equipes do Hospital resultaram em centenas de pesquisas desenvolvidas, na iniciação científica, nas dissertações de mestrado, teses de doutorado e de pós-doutorado, bem como no desenvolvimento de projetos de pesquisa nacionais e internacionais ou mesmo programas multicêntricos (RELATÓRIO DE GESTÃO, 2010-2014, p. 77). Importante destacar que no espaço do hospital tem estado envolvidos em estudos cerca de 800 pósgraduandos, inseridos em 151 linhas de pesquisa orientados por docentes da Faculdade de Ciências Médicas. No ano de 2013, foram produzidos 1.020 artigos da FCM publicados em periódicos nacionais e internacionais e 1.033 projetos de pesquisa apoiados por 94 laboratórios, dentro e fora da UNICAMP (ibid., p. 77).

Diante destes números e desta história, o HC se confirma como um hospital extremamente comprometido com a assistência, ensino e pesquisa e busca se tornar um dos melhores hospitais universitários de alta complexidade do Estado e do País, cem por

\footnotetext{
${ }^{2}$ Portal do HC UNICAMP, HC da UNICAMP comemora 30 anos focado no futuro. Disponível em: http://www.hc.unicamp.br/node/945.

Serv. Soc. \& Saúde, Campinas, SP v. 14, n. 2 (20), p. 149-154, jul./dez. 2015 ISSN 1676-6806
} 
cento público, para cuidar da vida como um todo, biológica, psíquica e social, dos pacientes que apresentem agravos da saúde.

Recebido em 15.11.2016 - Aprovado em 19.12.2016

\section{REFERÊNCIAS}

UNIVERSIDADE ESTADUAL DE CAMPINAS. Organização Geral [do] Hospital das Clínicas. Campinas. Maio/ 1977. Não-paginado.

UNIVERSIDADE ESTADUAL DE CAMPINAS. Portaria GR 062/86. Estrutura Administrativa do Hospital das Clínicas. 24 de março de 1986. Mimeografado. Nãopaginado.

UNIVERSIDADE ESTADUAL DE CAMPINAS. Novo Regimento do Hospital de Clínicas, deliberação do Conselho Universitário (CONSU), em 27 de setembro de 2006, publicada no Diário Oficial [do] Poder Executivo em 31 de outubro de 2006. HOSPITAL DE CLÍNICAS DA UNICAMP. Relatório de gestão 2010-2014. 89 p. Disponível: http://www.hc.unicamp.br/sites/default/files/users/Administrador/relatoriogestao-2010-2014.pdf PORTAL DO HC UNICAMP, HC da Unicamp comemora 30 anos focado no futuro. http://www.hc.unicamp.br/node/945 
Serv. Soc. \& Saúde, Campinas, SP v. 14, n. 2 (20), p. 149-154, jul./dez. 2015 ISSN 1676-6806 